Annali di Matematica pura ed applicata

(IV), Vol. CLXII (1992), pp. 215-226

\title{
GROUP VARIETIES RELATED TO THE KP HIERARCHY
}

\author{
FRANCESCO BOTTACIN
}

\begin{abstract}
Some remarkable relations between group varieties and the solutions of a physically important class of differential equations, called KP hierarchy, are found. In particular, it is proved that to each solution in a certain class, including a lot of physically important solutions such as the famous $n$-solitons, there is associated in a natural way a group variety.
\end{abstract}

\section{INTRODUCTION}

The purpose of this paper is to explain the connection existing between a wide class of group varieties and a physically important class of differential equations called KP hierarchy. It is a well known result of MUMford [10, vol. 2, Ch. IIIb, §5] that by using jacobian theta functions one can construct solutions to KP and KdV hierarchies. More precisely, he showed that starting from the jacobian theta function of a nonsingular curve one easily constructs special solutions to the KP hierarchy, and these functions satisfy also KdV hierarchy when the curve is hyperelliptic. Then, if one takes an algebraic family $C_{t}$ of smooth hyperelliptic curves of genus $n$, which tend to a singular curve $C_{0}$ as $t \rightarrow 0$, the jacobian varieties Jac $C_{t}$ of these curves tend to a generalized jacobian $\mathrm{Jac} C_{0}$, which is no longer an abelian variety but a more general group variety (it turns out to be a product of multiplicative groups). Under this deformation the solutions to the KdV hierarchy constructed from Riemann's theta function of Jac $C_{t}$ tend to another solution, which is just the famous $n$-soliton solution.

Conversely, KRICHEVER proved in [9] that quasi-periodic solutions of the KP hierarchy are essentially Riemann's theta functions of algebraic curves.

Here we will be interested in a much wider class of solutions of the KP hierarchy: to each element in this class corresponds a group variety. To show this we'll prove that all elements in this class are theta types: these are a generalization of classical theta functions introduced by BARsotTI in [1]. A theta type is essentially a nonzero element $\theta(x) \in k[[x]]$ such that $\theta(x+y) \theta(x-y) \in k[[x]] \otimes_{k} k[[y]]$, where $k$ is a field of characteristic zero. This last property implies that the field generated over $k$ by the logarithmic derivatives of $\theta(x)$, from the seconds on, is the function field of a group variety $V$ over $k$; moreover $\theta(x)$ determines a divisor of $V$, so that we get in effect a polarized group variety. A fundamental fact is that almost all group varieties can be constructed, with the method just described, starting from theta types. 
In conclusion, the solutions of the KP hierarchy we are considering here, being theta types, are naturally related to group varieties.

This paper is organized as follows: in Sect. 1 we recall how the condition $\theta(x+$ y) $\theta(x-y) \in k[[x]] \otimes_{k} k[[y]]$, defining theta types, can be translated in differential terms, in order to obtain the differential equations characterizing theta types. These are written in terms of certain "universal" polynomials with rational coefficients. Then we find the fundamental relations that link these polynomials with Hirota's differential operators. This is an easy but very important result because it shows that any differential equation which can be written by using Hirota's operators can be translated into a differential equation for theta types. If these equations are enough (in a sense that we'll make precise later) then their solutions are actually theta types. This argument applies not only to the KP hierarchy, as shown in this paper, but also to many other important classes of differential equations of mathematical physics, such as KdV hierarchy, Boussinesq hierarchy and so on (in fact, as already observed, it applies to all equations that admit an expression in terms of Hirota's operators).

In Sect. 2, after a brief description of the KP hierarchy, we prove that it is equivalent to a set of differential equations for theta types. Unfortunately, because of the complicated expression of these equations, we can't prove directly that their solutions are theta types, so, in Sect. 3, we develop a different approach to the problem. We introduce infinite order differential operators, called vertex operators, and prove firstly that they act infinitesimally on the space of $\tau$-functions, i.e. they transform a given solution of the KP hierarchy to another solution, and secondly that they also act on theta types, sending theta types to theta types. This finally proves that all $\tau$-functions which can be constructed by letting vertex operators act repeatedly on a $\tau$-function which is already known to be a theta type, are actually theta types. This is true, in particular, for $n$-solitons, which are constructed by the above method, starting from the constant function 1 .

\section{Preliminaries}

In this Section we recall some basic definitions and the construction of differential equations characterizing theta types. For a detailed treatment of the subject we refer the reader to [2], [3] and [4] ([3] generalizes the construction of differential equations to the case of several variables and corrects some errors occuring in [2]).

Let $k$ be an algebraically closed field of characteristic 0 and $k[[x]], x=\left(x_{1}, \ldots, x_{n}\right)$, the ring of formal power series in $n$ variables over $k$. A non-zero element $\theta(x) \in k[[x]]$ is a holomorphic theta type if it satisfies the following relation, called holomorphic prosthaferesis:

$$
\theta(x+y) \theta(x-y) \in k[[x]] \otimes_{k} k[[y]]
$$


If $k$ is the complex field a holomorphic theta type is actually an entire function (this is proved in [1] only for non degenerate thetas, but the same argument holds also for theta types).

Now we must introduce some notations. If $\mu=\left(\mu_{1}, \ldots, \mu_{n}\right), \nu=\left(\nu_{1}, \ldots, \nu_{n}\right) \in \mathbb{N}^{n}$ are multiindices and $r$ is a positive integer we let $\mu+\nu=\left(\mu_{1}+\nu_{1}, \ldots, \mu_{n}+\nu_{n}\right)$, $r \mu=\left(r \mu_{1}, \ldots, r \mu_{n}\right),|\mu|=\mu_{1}+\cdots+\mu_{n}$ and $\mu !=\mu_{1} ! \cdots \mu_{n} ! ; \mu \leq \nu$ means $\mu_{i} \leq \nu_{i}$ all $i$, and $\mu<\nu$ means $\mu_{i} \leq \nu_{i}$ but $\mu_{j}<\nu_{j}$ for some $j$. If $x=\left(x_{1}, \ldots, x_{n}\right), x^{\mu}$ means $x_{1}^{\mu_{1}} \cdots \cdots x_{n}^{\mu_{n}}$ and $D_{x}$, or simply $D$ if no confusion can arise, denotes derivation with respect to the variables $x$, more precisely we let:

$$
D_{x}^{\mu}=\frac{\partial^{|\mu|}}{\partial x_{1}^{\mu_{1}} \cdots \partial x_{n}^{\mu_{n}}} .
$$

Moreover, for a power series $\phi(x)$ and a multiindex $\mu>0$, we let $\phi_{\mu}(x)=$ $(\mu !)^{-1} D^{\mu} \log \phi(x)$. Using these notations we can write the following power series expansion

$$
\theta(x+y) \theta(x-y)=2 \theta^{2}(x) \sum_{\mu} P_{\mu}(\theta(x)) y^{\mu},
$$

the sum being over all $\mu \in \mathbb{N}^{n}$ such that $|\mu| \equiv 0 \bmod 2$. The $P_{\mu}$ 's are polynomial functions with rational coefficients in the $\theta_{\nu}$ 's, $0<\nu \leq \mu$, whose expressions are given by the following.

Lemma 1.3. - Let $\mu \in \mathbb{N}^{n}$ be a multiindex such that $|\mu| \equiv 0 \bmod 2$, and let $\nu_{1}, \ldots, \nu_{h} \in \mathbb{N}^{n}$ be all the multiindices such that $\left|\nu_{i}\right| \equiv 0 \bmod 2$ and $0<\nu_{i} \leq \mu$, $i=1, \ldots, h$. Then

$$
P_{\mu}(\theta(x))=\sum_{j} 2^{|j|-1}(j !)^{-1} \theta_{\nu_{1}}^{j_{1}} \cdots \theta_{\nu_{h}}^{j_{h}}
$$

where $j$ ranges over all $h$-tuples $j=\left(j_{1}, \ldots, j_{h}\right)$ of non-negative integers which satisfy $j_{1} \nu_{1}+\cdots+j_{h} \nu_{h}=\mu$.

For the proof of this result, see [2, Sect. 5] for functions of one variable, or [5, Ch. 2, Lemma 1.3] for the general case.

It is well known (cfr. [2] or [4, Theorem 3.2]) that a necessary and sufficient condition for a power series $\theta(x)$ to be a holomorphic theta type is that the $P_{\mu}(\theta)$ 's span a finite dimensional $k$-vector space; in other words only a finite number of such polynomials can be linearly independent. Note that a linear relation among the $P_{\mu}(\theta)$ 's is a nonlinear partial differential equation for the function $\theta(x)$, so we can say that holomorphic theta types are the solutions of appropriate systems of (infinite) nonlinear p.d.e.'s, written in terms of certain "universal" polynomials.

We only mention that this situation becomes much simpler if we consider power series in one argument; this is not really restrictive, since we know that almost all group varieties (precisely those whose possibly occuring vector direct factor has 
dimension $\leq 1$, hence all abelian varieties) can be "produced" by theta types in one variable.

Now we recall the definition of Hirota's bilinear differential operators. Let $P\left(Y_{1}, \ldots, Y_{n}\right) \in k\left[Y_{1}, \ldots, Y_{n}\right]$ be a polynomial and $\phi(x), \psi(x)$ functions. We define a differential operator as follows:

$$
P(D) \phi \cdot \psi=\left.P\left(\partial / \partial y_{1}, \ldots, \partial / \partial y_{n}\right)[\phi(x+y) \psi(x-y)]\right|_{y=0} .
$$

$P(D)$ is called Hirota's bilinear differential operator, and $P(D) \phi \cdot \psi=\zeta$ are Hirota's bilinear differential equations. See [8] for basic properties and applications of Hirota's operators.

In particular, for a power series $\theta(x) \in k[[x]]$ and a multiindex $\mu \in \mathbb{N}^{n}$, we have

$$
\left(D^{\mu} \theta \cdot \theta\right)(x)=\left.D_{y}^{\mu}[\theta(x+y) \theta(x-y)]\right|_{y=0},
$$

thus we can write the following Taylor expansion:

$$
\theta(x+y) \theta(x-y)=\sum_{\mu}(\mu !)^{-1}\left(D^{\mu} \theta \cdot \theta\right)(x) y^{\mu},
$$

where the sum is over all $\mu \in \mathbb{N}^{n}$, such that $|\mu| \equiv 0 \bmod 2$ (it follows immediately from the definition that $D^{\mu} \theta \cdot \theta$ is identically zero if $|\mu|$ is odd).

Comparing (1.4) with (1.2), we find that

$$
\left(D^{\mu} \theta \cdot \theta\right)(x)=2 \mu ! \theta^{2}(x) P_{\mu}(\theta(x)), \quad|\mu| \equiv 0 \quad \bmod 2 .
$$

This is the fundamental relation that links Hirota's operators with the universal polynomials, and it shows that all differential equations for theta types can also be written in the bilinear form of Hirota.

\section{The KP hierarchy And its CONnections With theta types}

Let us denote by $x$ the infinite set of variables $\left(x_{1}, x_{2}, \ldots, x_{n}, \ldots\right)$ and by $\partial$ the derivation $\partial / \partial x_{1}$. A formal pseudodifferential operator $P$ is a formal Laurent series in $\partial^{-1}$ with coefficients in $k[[x]]$ :

$$
P=\sum_{j=-\infty}^{m} a_{j}(x) \partial^{j}, \quad m \in \mathbb{Z}, \quad a_{j}(x) \in k[[x]] .
$$

If $a_{m}(x) \neq 0, m$ will be called the order of $P$, in symbols $m=\operatorname{ord} P$.

If we define the product of two pseudodifferential operators by the following generalization of the Leibniz rule

$$
\left(\sum_{i} a_{i}(x) \partial^{i}\right)\left(\sum_{j} b_{j}(x) \partial^{j}\right)=\sum_{\substack{i, j \\
h \geq 0}}\left(\begin{array}{l}
j \\
h
\end{array}\right) a_{i}(x)\left(\partial^{h} b_{j}\right)(x) \partial^{i+j-h}
$$

it follows that the set of all formal pseudodifferential operators $\Psi=k[[x]]\left(\left(\partial^{-1}\right)\right)$ is a non-commutative $k$-algebra and is a Lie algebra under commutation of operators. 
We denote by $\Psi^{-}$and $\Psi^{+}$the Lie subalgebras of $\Psi$ consisting of operators of order $\leq-1$ and of ordinary differential operators respectively. We have $\Psi=\Psi^{-} \oplus \Psi^{+}$ as a $k$-vector space, and for $P \in \Psi$, we denote the corresponding decomposition by $P=P_{-}+P_{+}$. We also introduce the space of formal oscillating functions

$$
\left\{f(x, \lambda) \exp \xi(x, \lambda) \mid f(x, \lambda) \in k[[x]]\left(\left(\lambda^{-1}\right)\right)\right\},
$$

where $\lambda$ is a formal parameter and $\xi(x, \lambda)=\sum_{i=1}^{\infty} x_{i} \lambda^{i}$.

The $k$-algebra $\Psi$ acts in an obvious way on this space of functions.

Now we consider the following isospectral deformation problem (see [7, Sect. 1]):

$$
\left\{\begin{array}{l}
L w=\lambda w, \\
\frac{\partial w}{\partial x_{n}}=B_{n} w, \quad n=1,2, \ldots,
\end{array}\right.
$$

where $L$ is a pseudodifferential operator of the form $L=L(x)=\partial+u_{-1}(x) \partial^{-1}+$ $u_{-2}(x) \partial^{-2}+\ldots, B_{n}=\left(L^{n}\right)_{+}$and $w$ is a formal oscillating function

$$
w(x, \lambda)=\left(\sum_{i=0}^{\infty} w_{i}(x) \lambda^{-i}\right) \exp \xi(x, \lambda),
$$

normalized by letting $w_{0}(x)=1$. Such a function $w(x, \lambda)$, satisfying $(2.1)$, is called a wave function for the operator $L$.

The integrability conditions of (2.1) constitute an infinite system of differential equations for the operator $L$, called the KP hierarchy:

$$
\frac{\partial L}{\partial x_{n}}=\left[B_{n}, L\right], \quad n=1,2, \ldots
$$

It can be shown (see, for example, [11, Sect. 1.2]) that the system (2.1) has a solution $w$ if and only if $L$ satisfies the KP hierarchy (2.2), in which case the solution is unique up to multiplication by elements of $1+k\left[\left[\lambda^{-1}\right]\right] \lambda^{-1}$.

Let then $w(x, \lambda)$ be a wave function for the operator $L$. It is shown in $[7$, Sect. 1] that there exists a power series $\tau(x) \in k[[x]]$, called the $\tau$-function associated to $L$, such that

$$
w(x, \lambda)=\frac{\tau\left(x_{1}-1 / \lambda, x_{2}-1 / 2 \lambda^{2}, x_{3}-1 / 3 \lambda^{3}, \ldots\right)}{\tau(x)} \exp \xi(x, \lambda) .
$$

Moreover the KP hierarchy can be written in terms of $\tau$-functions as an infinite set of Hirota's bilinear differential equations, given by the following generating function expansion:

$$
\sum_{i=0}^{\infty} p_{i}(-2 y) p_{i+1}(\tilde{D}) \exp \left(\sum_{j=1}^{\infty} y_{j} D_{j}\right) \tau \cdot \tau=0
$$


where $p_{n}(x)$ are Schur polynomials, defined by

$$
\exp \left(\sum_{j=1}^{\infty} x_{j} q^{j}\right)=\sum_{n=0}^{\infty} p_{n}(x) q^{n}
$$

and $\tilde{D}=\left(D_{1}, 2^{-1} D_{2}, 3^{-1} D_{3}, \ldots\right)$ with the $D_{j}$ 's being Hirota's symbols, so that for each multiindex $\mu$, the coefficient of $y^{\mu}$ in (2.4) gives Hirota's equation for a $\tau$-function.

In the preceding section we have found the relations (1.5) between Hirota's bilinear differential operators and Barsotti's universal polynomials, so it is now immediate to see that the KP hierarchy, written in terms of $\tau$-functions, is equivalent to an infinite set of linear relations for polynomials $P_{\mu}(\tau(x))$. The calculations can be effectively worked out, as shown in the author's thesis [5], by finding an explicit expression for the equations of the KP hierarchy (2.4) and then by replacing each $D^{\mu} \tau \cdot \tau$ with the corresponding expression given by (1.5). This leads to a rather complicated but explicit formulation of the linear relations among the universal polynomials. Precisely, for every multiindex $\beta \in \mathbb{N}^{\infty}$, we have the following linear relation $[5$, Ch. 2, 4.7]:

$$
\sum_{\substack{\alpha \in \mathbb{N}^{\infty} \\|\alpha|^{*}=|\beta|^{*}+1 \\|\alpha|=0 \bmod 2}} \frac{\alpha !}{\mathbf{1}^{\alpha}}\left(\sum_{\substack{\mu \in \mathbb{N}^{\infty} \\ \beta-\alpha \leq \mu \leq \beta}} \frac{(-2)^{|\mu|}}{\mathbf{1}^{\mu} \mu !(\alpha+\mu-\beta) !(\beta-\mu) !}\right) P_{\alpha}(\tau(x))=0,
$$

where $|\mu|^{*}=\mu_{1}+2 \mu_{2}+3 \mu_{3}+\cdots$, and $\mathbf{1}^{\mu}=1^{\mu_{1}} \cdot 2^{\mu_{2}} \cdot 3^{\mu_{3}} \cdots$.

As an example, the choices $\beta=(3,0,0, \ldots), \beta=(1,1,0,0, \ldots)$ or $\beta=$ $(0,0,1,0, \ldots)$ all give

$$
12 P_{(4,0,0)}+3 P_{(0,2,0)}-2 P_{(1,0,1)}=0
$$

which is the first nontrivial relation among the $P_{\mu}$ 's (cited also in [4, Theorem 3.5]) and which corresponds to the first equation of the KP hierarchy, namely the classical $\mathrm{KP}$ equation in Hirota's form:

$$
\left(D_{1}^{4}+3 D_{2}^{2}-4 D_{1} D_{3}\right) \tau \cdot \tau=0 .
$$

Unfortunately it is still unknown whether the relations given by (2.5) are enough to ensure that the $k$-vector space spanned by the $P_{\mu}$ 's has finite dimension. If this is the case then this argument proves that all $\mathrm{KP} \tau$-functions are theta types (for the sake of definiteness when we speak of theta types we have to consider power series in a finite number of variables, hence we must consider $\tau$-functions for the KP hierarchy which satisfy the extra condition of depending on finitely many variables).

In the particular case of functions of one variable, (2.5) reduces to

$$
\left(\sum_{i=0}^{n} \frac{(-2)^{i}}{i !(i+1) !(n-i) !}\right) P_{n+1}(\tau(x))=0, \quad \forall n \in \mathbb{N}, \quad n \equiv 1 \quad \bmod 2
$$


Since it is not difficult to prove that the coefficient of $P_{n+1}$ in $(2.6)$ is $\neq 0$ for every integer $n>1$ (see [5, Ch. 2, Lemma 4.9]), this shows that the polynomials $P_{n}(\tau(x)$ ) span a vector space of dimension 1 , hence all $\tau$-functions of one variable are actually theta types.

We only mention here that, by the analysis of some numerical computations, we are led to believe that the same conclusion holds also for $\mathrm{KP} \tau$-functions depending on finitely many variables (see [5, Ch. 2, Sect. 4]).

\section{Soliton solutions of The KP Hierarchy}

In this section we will prove that a particular class of solutions of the KP hierarchy, namely the soliton solutions, are theta types. This result will be achieved by using a quite different approach to the problem from the one developed in the preceding section.

First we start with an observation. Let $f(t)$ be a function of a single variable $t$ and $d / d t$ the usual derivation. By considering the formal Taylor expansion of $\exp (\lambda d / d t)$, it is easy to see that this operator acts by translation on $t$ :

$$
\exp \left(\lambda \frac{d}{d t}\right) f(t)=f(t+\lambda) \text {. }
$$

Now we introduce the following infinite order differential operator, called a vertex operator:

$$
X_{x}(\lambda)=\exp (\xi(x, \lambda)) \exp \left(-\xi\left(\tilde{\partial}, \lambda^{-1}\right)\right),
$$

where $\tilde{\partial}=\left(\partial / \partial x_{1}, 2^{-1} \partial / \partial x_{2}, 3^{-1} \partial / \partial x_{3}, \ldots\right)$.

Since two operators of the type $\exp \left(a_{i} \partial / \partial x_{i}\right)$ and $\exp \left(a_{j} \partial / \partial x_{j}\right)$ commute, we have

$$
\exp \left(-\xi\left(\tilde{\partial}, \lambda^{-1}\right)\right)=\prod_{i=1}^{\infty} \exp \left(-\frac{1}{i \lambda^{i}} \frac{\partial}{\partial x_{i}}\right)
$$

hence

$$
\exp \left(-\xi\left(\tilde{\partial}, \lambda^{-1}\right)\right) f\left(x_{1}, x_{2}, \ldots\right)=f\left(x_{1}-\frac{1}{\lambda}, x_{2}-\frac{1}{2 \lambda^{2}}, x_{3}-\frac{1}{3 \lambda^{3}}, \ldots\right) .
$$

In view of this last relation, (3.1) and (2.3), we deduce that

$$
w(x, \lambda)=\frac{X_{x}(\lambda) \tau(x)}{\tau(x)}
$$

where $w$ is the wave function associated to the $\tau$-function $\tau(x)$.

Analogously, if we consider the operator

$$
X_{x}^{*}(\lambda)=\exp (-\xi(x, \lambda)) \exp \left(\xi\left(\tilde{\partial}, \lambda^{-1}\right)\right),
$$

we have

$$
w^{*}(x, \lambda)=\frac{\tau\left(x_{1}+1 / \lambda, x_{2}+1 / 2 \lambda^{2}, \ldots\right)}{\tau(x)} \exp (-\xi(x, \lambda))=\frac{X_{x}^{*}(\lambda) \tau(x)}{\tau(x)} .
$$


$w^{*}(x, \lambda)$ is called the adjoint wave function for the KP hierarchy.

A fundamental result, proved in [7, Sect. 1], states that the wave function and the adjoint wave function for the KP hierarchy are characterized by the following bilinear identity:

$$
\operatorname{Res}_{\lambda=\infty} w(x, \lambda) w^{*}(y, \lambda) d \lambda=0, \quad \forall x, y .
$$

For the sake of simplicity we introduce here the following notations:

$$
\begin{aligned}
& G_{x}(\lambda) \tau(x)=\tau\left(x_{1}-\frac{1}{\lambda}, x_{2}-\frac{1}{2 \lambda^{2}}, \ldots\right), \\
& G_{x}^{*}(\lambda) \tau(x)=\tau\left(x_{1}+\frac{1}{\lambda}, x_{2}+\frac{1}{2 \lambda^{2}}, \ldots\right),
\end{aligned}
$$

so that we have

$$
\begin{aligned}
& X_{x}(\lambda) \tau(x)=\exp (\xi(x, \lambda)) G_{x}(\lambda) \tau(x), \\
& X_{x}^{*}(\lambda) \tau(x)=\exp (-\xi(x, \lambda)) G_{x}^{*}(\lambda) \tau(x) .
\end{aligned}
$$

Now, using (3.2), (3.4) and (3.7), we can rewrite the bilinear identity (3.5) for $\tau$ functions as follows:

$$
\operatorname{Res}_{\lambda=\infty}\left(G_{x}(\lambda) \tau(x)\right)\left(G_{y}^{*}(\lambda) \tau(y)\right) \exp (\xi(x-y, \lambda)) d \lambda=0, \quad \forall x, y .
$$

This last relation is completely equivalent to the KP hierarchy (2.4).

Now we introduce another vertex operator, depending on two formal parameters $p$ and $q$ :

$$
X_{x}(p, q)=\exp (\xi(x, p)-\xi(x, q)) \exp \left(-\xi\left(\tilde{\partial}, p^{-1}\right)+\xi\left(\tilde{\partial}, q^{-1}\right)\right) .
$$

We claim that this operator acts infinitesimally on the space of $\tau$-functions of the KP hierarchy:

Proposition 3.10. - If $\tau(x)$ satisfies (3.8), the same holds also for $X_{x}(p, q) \tau(x)$.

Proof. - With the preceding notations, we have

$$
X_{x}(p, q) \tau(x)=\exp (\xi(x, p)-\xi(x, q)) G_{x}(p) G_{x}^{*}(q) \tau(x) .
$$

So let us start with a $\tau$-function $\tau(x)$. First we compute

$$
\begin{aligned}
& G_{x}(\lambda) X_{x}(p, q) \tau(x)=G_{x}(\lambda)\left[\exp (\xi(x, p)-\xi(x, q)) G_{x}(p) G_{x}^{*}(q) \tau(x)\right]= \\
& \quad=\exp \left(\sum_{i=1}^{\infty}\left(x_{i}-1 / i \lambda^{i}\right) p^{i}-\sum_{i=1}^{\infty}\left(x_{i}-1 / i \lambda^{i}\right) q^{i}\right) G_{x}(\lambda) G_{x}(p) G_{x}^{*}(q) \tau(x),
\end{aligned}
$$

and

$$
\begin{aligned}
& G_{y}^{*}(\lambda) X_{y}(p, q) \tau(y)=G_{y}^{*}(\lambda)\left[\exp (\xi(y, p)-\xi(y, q)) G_{y}(p) G_{y}^{*}(q) \tau(y)\right]= \\
& \quad=\exp \left(\sum_{i=1}^{\infty}\left(y_{i}+1 / i \lambda^{i}\right) p^{i}-\sum_{i=1}^{\infty}\left(y_{i}+1 / i \lambda^{i}\right) q^{i}\right) G_{y}^{*}(\lambda) G_{y}(p) G_{y}^{*}(q) \tau(y)
\end{aligned}
$$


From these we derive:

$$
\begin{aligned}
& \operatorname{Res}_{\lambda=\infty}\left(G_{x}(\lambda) X_{x}(p, q) \tau(x)\right)\left(G_{y}^{*}(\lambda) X_{y}(p, q) \tau(y)\right) \exp (\xi(x-y, \lambda)) d \lambda= \\
&= \operatorname{Res}_{\lambda=\infty} \exp (\xi(x, p)-\xi(x, q)+\xi(y, p)-\xi(y, q))\left(G_{x}(\lambda) G_{x}(p) G_{x}^{*}(q) \tau(x)\right) . \\
& \cdot\left(G_{y}^{*}(\lambda) G_{y}(p) G_{y}^{*}(q) \tau(y)\right) \exp (\xi(x-y, \lambda)) d \lambda= \\
&=\exp (\xi(x, p)-\xi(x, q)+\xi(y, p)-\xi(y, q)) G_{x}(p) G_{x}^{*}(q) G_{y}(p) G_{y}^{*}(q) . \\
& \cdot \operatorname{Res}_{\lambda=\infty}\left(G_{x}(\lambda) \tau(x)\right)\left(G_{y}^{*}(\lambda) \tau(y)\right) \exp (\xi(x-y, \lambda)) d \lambda=0 .
\end{aligned}
$$

This is what we wished to prove. Q.E.D.

This result provides a good method for constructing $\tau$-functions. To obtain a large class of solutions we just let the operators $X(p, q)$ 's act repeatedly on the constant function 1, which obviously solves the KP hierarchy. Among the $\tau$-functions so constructed there are some of particular interest, namely the so-called $n$-solitons, defined as follows:

$$
\tau\left(x ; a_{1}, p_{1}, q_{1} ; \ldots ; a_{n}, p_{n}, q_{n}\right)=\exp \left(\sum_{j=1}^{n} a_{j} X_{x}\left(p_{j}, q_{j}\right)\right) 1 .
$$

For a detailed description of the full power of this approach to the study of $\tau$ functions of the KP hierarchy we refer the reader to [7] and, in particular, to [6].

From now on we let $x$ denote finitely many variables $\left(x_{1}, \ldots, x_{n}\right)$ (we make this restriction because we want to speak of theta types, but analogous results also hold for infinitely many variables). If $f(x) \in k[[x]]$, it follows immediately from standard arguments on formal power series that $G_{x}(\lambda) f(x)$ and $G_{x}^{*}(\lambda) f(x)$ belong to $k\left[\left[\lambda^{-1}\right]\right][[x]]$. Having this result in hand, we now claim that $X_{x}(\lambda) f(x) \in$ $k\left(\left(\lambda^{-1}\right)\right)[[x]]$, where $k\left(\left(\lambda^{-1}\right)\right)$ denotes the ring of formal Laurent series in $\lambda^{-1}$. To prove this we need the following

Lemma 3.12. - Let $\xi(x, \lambda)=\sum_{i=1}^{n} x_{i} \lambda^{i}$. Then we have the following expansion:

$$
\exp (\xi(x, \lambda))=\sum_{\mu \in \mathbb{N}^{n}} \frac{x^{\mu}}{\mu !} \lambda^{|\mu|^{*}}
$$

where $|\mu|^{*}=\mu_{1}+2 \mu_{2}+\cdots+n \mu_{n}$.

We omit the proof of this fact since it is straightforward.

Now we can prove the assertion preceding Lemma 3.12:

Proposition 3.13. - If $f(x) \in k[[x]]$, then $X_{x}(\lambda) f(x) \in k\left(\left(\lambda^{-1}\right)\right)[[x]]$.

Proof. - We have already observed that $G_{x}(\lambda) f(x) \in k\left[\left[\lambda^{-1}\right]\right][[x]]$, so let

$$
G_{x}(\lambda) f(x)=\sum_{\nu \in \mathbb{N}^{n}} a_{\nu}\left(\lambda^{-1}\right) x^{\nu}
$$

where $a_{\nu}\left(\lambda^{-1}\right) \in k\left[\left[\lambda^{-1}\right]\right]$. 
Now, by the preceding Lemma, we have:

$$
\begin{array}{r}
X_{x}(\lambda) f(x)=\exp (\xi(x, \lambda)) G_{x}(\lambda) f(x)=\left(\sum_{\mu \in \mathbb{N}^{n}} \frac{x^{\mu}}{\mu !} \lambda^{|\mu|^{*}}\right)\left(\sum_{\nu \in \mathbb{N}^{n}} a_{\nu}\left(\lambda^{-1}\right) x^{\nu}\right)= \\
=\sum_{\mu, \nu} \frac{a_{\nu}\left(\lambda^{-1}\right) \lambda^{|\mu|^{*}}}{\mu !} x^{\mu+\nu} .
\end{array}
$$

If we set $\alpha=\mu+\nu$, we can rewrite the last expression as follows:

$$
\sum_{\alpha \in \mathbb{N}^{n}}\left(\sum_{0 \leq \mu \leq \alpha} \frac{a_{\alpha-\mu}\left(\lambda^{-1}\right) \lambda^{|\mu|^{*}}}{\mu !}\right) x^{\alpha} .
$$

This is what we sought to prove, since the coefficient of $x^{\alpha}$ is expressed as a finite sum of elements in $k\left(\left(\lambda^{-1}\right)\right)$. Q.E.D.

Let us consider the quotient field $K=Q\left(k\left(\left(\lambda^{-1}\right)\right)\right)$ : in view of the preceding Proposition we have a function $X_{x}(\lambda): k[[x]] \rightarrow K[[x]]$. The fundamental fact is that this vertex operator sends theta types to theta types. More precisely we have:

TheOREm 3.14. - Let $\theta(x) \in k[[x]]$ be a holomorphic theta type. Then $X_{x}(\lambda) \theta(x)$ is a holomorphic theta type defined over the field $K=Q\left(k\left(\left(\lambda^{-1}\right)\right)\right)$.

Proof. - By definition $\theta(x)$ satisfies the holomorphic prosthaferesis

$$
\theta(x+y) \theta(x-y) \in k[[x]] \otimes_{k} k[[y]] .
$$

If we apply $G_{x}(\lambda)$ to this relation, we get:

$$
\begin{aligned}
\left(G_{x}(\lambda) \theta\right)(x+y)\left(G_{x}(\lambda) \theta\right)(x-y) \in G_{x}(\lambda) k[[x]] \otimes_{k} k[[y]] & \\
\hookrightarrow K[[x]] \otimes_{k} k[[y]] & \hookrightarrow[[x]] \otimes_{K} K[[y]] .
\end{aligned}
$$

Moreover we note that $\exp (\xi(x, \lambda)) \in K[[x]]$, so that finally we have:

$$
\begin{aligned}
& \left(X_{x}(\lambda) \theta\right)(x+y)\left(X_{x}(\lambda) \theta\right)(x-y)= \\
& \quad=\exp (\xi(x+y, \lambda))\left(G_{x}(\lambda) \theta\right)(x+y) \exp (\xi(x-y, \lambda))\left(G_{x}(\lambda) \theta\right)(x-y)= \\
& \quad=\exp (2 \xi(x, \lambda))\left(G_{x}(\lambda) \theta\right)(x+y)\left(G_{x}(\lambda) \theta\right)(x-y) \in K[[x]] \otimes_{K} K[[y]] . \quad \text { Q.E.D. }
\end{aligned}
$$

It is now immediate to see that the same conclusion also holds for vertex operators of the form $X(p, q)$, after setting $K=Q\left(k\left(\left(p^{-1}, q^{-1}\right)\right)\right)$.

This finally proves that all $\tau$-functions of the KP hierarchy (with the restriction on the number of variables stated before) which can be obtained by the action of vertex operators on the constant function 1, for instance, or, more generally, on $\tau$ functions which are already known to be theta types (polynomial or quasi-periodic $\tau$-functions, for example), are actually holomorphic theta types. In particular this is true for soliton solutions of the KP hierarchy, as claimed before.

To end this Section we mention that the situation described above gets better if we take $k$ to be the complex field. In fact, holomorphic theta types on the complex 
field are actually entire functions, hence $G_{x}(\lambda) \theta(x)$ is a convergent power series for every $x \in \mathbb{C}$ and $\lambda \in \mathbb{C}-\{0\}$. This proves that $X_{x}(\lambda) \theta(x) \in \mathbb{C}[[x]]$ for every $\lambda \in \mathbb{C}-\{0\}$, hence we can again take the complex field as the field $K$. So vertex operators send theta types defined over the complex field to theta types over the complex field.

\section{Note added.}

The result we have proved in Sect. 3 does not apply immediately to the special case of solitons, since this requires the use of differential operators of the form $\exp (a X(p, q))$, instead of the simpler $X(p, q)$ 's. However it is true that the $n$-soliton $\tau$-functions are theta types, as may be shown in a direct way, using an explicit expression for these functions and the prosthaferesis formula. We shall come on this in a forthcoming paper.

\section{REFERENCES}

[1] I. Barsotti, Considerazioni sulle funzioni theta, Symp. Math., 3 (1970), p. 247.

[2] I. Barsotti, Le equazioni differenziali delle funzioni theta, Rend. Acc. Naz. XL, 101 (1983), p. 227.

[3] I. Barsotti, Le equazioni differenziali delle funzioni theta; continuazione, Rend. Acc. Naz. XL, 103 (1985), p. 215.

[4] I. Barsotti, A new look for thetas, Proc. Symp. Pure Math., 47 (1987).

[5] F. Bottacin, Metodi algebrici nella teoria delle equazioni differenziali delle funzioni theta, Tesi dell'Università di Padova (1987-88).

[6] E. Date - M. Jimbo - M. Kashiwara - T. MiWA, Transformation groups for soliton equations - Euclidean Lie algebras and reduction of the KP hiererchy, Publ. RIMS, Kyoto Univ., 18 (1982), p. 1077.

[7] E. Date - M. Jimbo - M. Kashiwara - T. Miwa, Transformation groups for soliton equations, in Proceedings RIMS Symp. Nonlinear Integrable Systems-Classical Theory and Quantum Theory (Kyoto 1981), p. 39, World Scientific, Singapore (1983).

[8] R. Hirota, Direct method of finding exact solutions of nonlinear evolution equations, Lect. Notes Math., 515, pp. 40-68, Springer, Berlin-Heidelberg-New York (1976).

[9] I.M. KRICHEVER, Methods of algebraic geometry in the theory of nonlinear equations, Russ. Math. Surv., 32 (1977), p. 185.

[10] D. Mumford, Tata Lectures on Theta, Birkhäuser, Boston-Basel-Stuttgart, vol. 1 (1983), vol. 2 (1984).

[11] T. Shiota, Characterization of Jacobian varieties in terms of soliton equations, Invent. Math., 83 (1986), p. 333.

Dipartimento di Matematica Pura e Applicata, Università degli Studi di Padova, Via Belzoni 7, 35131 Padova, Italy

E-mail address: bottacin@math.unipd.it 